\title{
EDITORIAL
}

\section{Innocent white blood cells in sporadic Creutzfeldt-Jakob disease?}

\author{
Soon-Tae Lee and Jae-Kyu Roh \\ Laboratory Investigation (2009) 89, 612-613; doi:10.1038/labinvest.2009.42
}

\section{A} fter several cases of prion transmission were reported in patients who received non-leukodepleted blood products associated with variant CreutzfeldtJakob disease (vCJD), ${ }^{1}$ highthroughput and reliable detection methods for prion-tainted blood units became necessary. As white blood cells (WBCs) in blood buffy coat are infectious in $\mathrm{vCJD}^{2}$ and leukodepletion reduces $\mathrm{WBC}$-associated prion infectivity, ${ }^{3}$ many countries have implemented leukodepletion. Although it has been believed that the potential of leukodepletion for prion reduction might offset its high cost, it is still debatable whether universal leukodepletion is sufficient, or even necessary for protecting transfusion recipients from CJD prions. Leukodepletion removes only about $42 \%$ of the total prion infectivity in endogenously infected blood. ${ }^{3}$ Prion infectivity in the peripheral blood of experimental animal models is present in all blood components including WBC, platelets and plasma. ${ }^{4}$ Given the volumes, roughly equal amounts of infectivity are found in WBC and soluble plasma fractions. Accordingly, leukodepletion alone is likely to be insufficient for the protection against prion transmission in VCJD.

Although more than 200 cases of vCJD have been reported so far, the rise of vCJD cases has leveled off along with resolution of the bovine spongiform encephalopathy crisis. However, sporadic CJD (sCJD) still occurs worldwide at a rate of about 1 case per million population every year. ${ }^{5,6}$ Figure 1 shows the classic histological triad of CJD: neuronal loss, spongiform change and gliosis. In this issue of Laboratory Investigation, Choi et $a l^{7}$ reported the levels of normal prion protein $\left(\operatorname{PrP}^{c}\right)$ and the disease-causing isoform $\left(\mathrm{PrP}^{\mathrm{Sc}}\right)$ of each WBC subpopulation in sCJD patients and control participants. $\operatorname{PrP}^{\mathrm{c}}$ was widely detected among WBC subpopulations, and the highest levels of $\operatorname{PrP}^{\mathrm{c}}$ were found in effector memory T cells. However, neither WBC composition nor the amount of cell-surface $\mathrm{PrP}^{\mathrm{c}}$ molecules was altered in SCJD patients. In $\mathrm{PrP}^{\mathrm{Sc}}$ analysis, they detected no evidence of $\operatorname{PrP}^{\mathrm{Sc}}$ in
Department of Neurology, Seoul National University Hospital, Seoul, South Korea. Correspondence should be addressed to: rohjk@snu.ac.kr

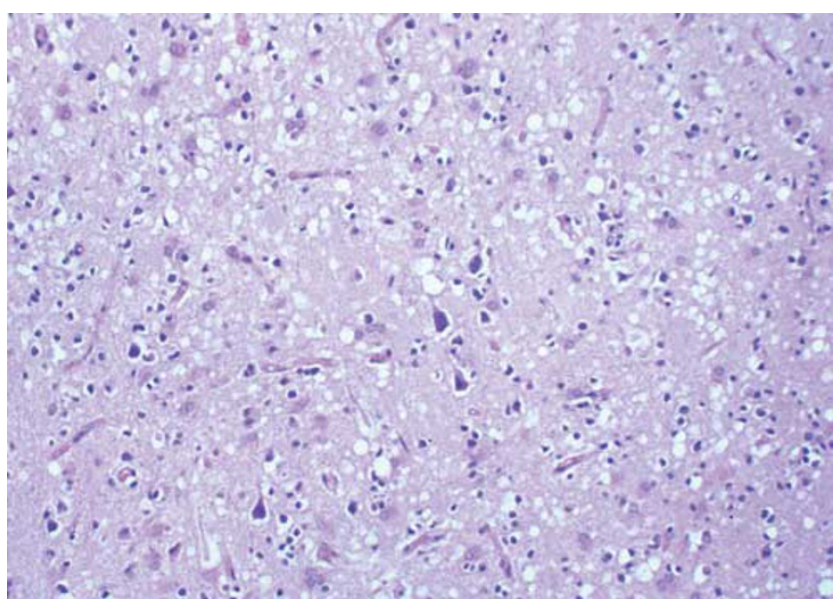

Figure 1 Hematoxylin and eosin-stained tissue section of sporadic Creutzfeldt-Jakob disease showing the classic histological triad of neuronal loss, spongiform change and gliosis. Photo courtesy of Anthony T Yachnis, MD, University of Florida. 
WBC and platelets. Although inoculation studies using WBCs from sCJD patients in animal models were not conducted, the authors concluded that WBCs of sCJD patients harbor low infectivity at worst.

As prions can be present in the plasma fraction, the possibility of sCJD prion transmission through transfusion of plasma still exists. Nevertheless, this study casts doubt on the necessity of leukodepletion to prevent prion transmission from sCJD patients. All of these concerns are still theoretical because there has not yet been a SCJD case associated with blood transfusion. However, it is not appropriate to overestimate the risk of sCJD transmission by blood transfusion. The issue of prion transmission by transfusion has been largely studied in vCJD models so far, and the spectrum of tissues and biosamples harboring infectivity partly depends on the strain of prions. Accordingly, the study from Choi et al not only suggests a re-evaluation of the practice of leukodepletion, but also lays the groundwork for a new model of blood-born transmission issue of sCJD.

1. Llewelyn $C A$, Hewitt $P E$, Knight $R S$, et al. Possible transmission of variant Creutzfeldt-Jakob disease by blood transfusion. Lancet 2004;363:417-421.

2. Bons $\mathrm{N}$, Lehmann $\mathrm{S}$, Mestre-Francès $\mathrm{N}$, et al. Brain and buffy coat transmission of bovine spongiform encephalopathy to the primate Microcebus murinus. Transfusion 2002;42:513-516.

3. Gregori L, McCombie N, Palmer D, et al. Effectiveness of leucoreduction for removal of infectivity of transmissible spongiform encephalopathies from blood. Lancet 2004;364:529-531.

4. Cervenakova L, Yakovleva O, McKenzie C, et al. Similar levels of infectivity in the blood of mice infected with humanderived VCJD and GSS strains of transmissible spongiform encephalopathy. Transfusion 2003;43:1687-1694.

5. Allen CT, Sonnen J, Leslie MJ, et al. Washington statewide pathology surveillance for prion disease. Ann Neurol 2007;61:371-372.

6. Ladogana A, Puopolo M, Croes EA, et al. Mortality from Creutzfeldt-Jakob disease and related disorders in Europe, Australia, and Canada. Neurology 2005;64:1586-1591.

7. Choi EM, Geschwind MD, Deering $C$, et al. Prion proteins in subpopulation of white blood cells from patients with sporadic Creutzfeldt-Jakob disease. Lab Invest 2009;89:624-635 (this issue). 